ARTICLE

\title{
Chemical engineering of quasicrystal approximants in lanthanide-based coordination solids
}

\author{
Laura Voigt (10 ${ }^{1}$, Mariusz Kubus ${ }^{1} \&$ Kasper S. Pedersen (iD) ${ }^{1 凶}$
}

Tessellation of self-assembling molecular building blocks is a promising strategy to design metal-organic materials exhibiting geometrical frustration and ensuing frustrated physical properties. Appearing in two-dimensional quasiperiodic phases, tilings consisting of fivevertex nodes are regarded as approximants for quasicrystals. Unfortunately, these structural motifs are exceedingly rare due to the complications of acquiring five-fold coordination confined to the plane. Lanthanide ions display the sufficient coordinative plasticity, and large ionic radii, to allow their incorporation into irregular molecule-based arrays. We herein present the use of ytterbium(II) as a five-vertex node in a two-dimensional coordination solid, $\mathrm{Ybl}_{2}\left(4,4^{\prime} \text {-bipyridine }\right)_{2.5}$. The semi-regular Archimedean tessellation structure verges on quasicrystallinity and paves the way for lanthanide-based metal-organic materials with interesting photonic and magnetic properties.

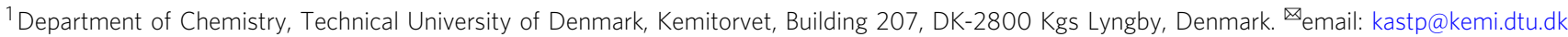


O uasicrystals are ordered materials lacking translational symmetry $^{1}$, which leads to unique physical properties ${ }^{2}$, including novel magnetic and photonic phenomena ${ }^{3-5}$. They exhibit symmetries that are forbidden for any periodic structure, such as the 5-fold rotational symmetry first discovered in an Al-Mn alloy by Shechtman et al. ${ }^{6}$ or the 12 -fold symmetry in dodecagonal quasicrystals ${ }^{7}$. Dodecagonal symmetry is achieved in a maximally random assembly of squares and triangles linked through four-, five-, and six-vertex nodes ${ }^{8,9}$. Dodecagonal quasicrystallinity has been observed in a plethora of materials including alloys ${ }^{10,11}$, mesoporous silica ${ }^{12}$, nanoparticle superlattices $^{13}$, and copolymers ${ }^{14}$. Notably, all of these quasicrystalline phases have motifs of, or coexist with, domains of periodic tilings of squares and triangles, whose existence was hypothesized by Frank and Kasper ${ }^{15}$, and whose topologies were first introduced by Kepler in Harmonices Mundi in $1619^{16}$. The tilings of squares and triangles belong to the uniform tessellations of the twodimensional (2D) (Euclidian) plane ${ }^{17}$, where all vertices are identical and the tilings are labeled by the sequence of their surrounding polygons. For instance, six-vertex nodes are found only in the regular triangular tiling $\left(3^{6}\right.$; Fig. 1a) containing a single equilateral polygon, whereas five-vertex nodes are part of the snub hexagonal $\left(3^{4} .6\right)$, elongated triangular $\left(3^{3} .4^{2}\right)$, and snub square tilings $\left(3^{2} .4 .3 .4\right.$; cf. Fig. $\left.1 \mathrm{~b}, \mathrm{e}, \mathrm{f}\right)$. Due to their similarities with the local structure of dodecagonal quasicrystals, crystalline phases with structure motifs of five-vertex semi-regular Archimedean tessellations (ATs) are referred to as quasicrystal approximants $9,12,18-20$. The appearance of approximants suggests the possibility of designing quasicrystallinity by chemical means. The realization of regular tilings in chemistry and nature is ubiquitous. However, of the ATs only the trihexagonal tiling $\left((3.6)^{2}\right.$; cf. Fig. 1c), the kagomé lattice, appears frequently ${ }^{21}$. Notably, ATs in materials have been hailed as a key component for exploring novel photonics applications ${ }^{22-24}$ and to host peculiar magnetic phenomena, such as frustration ${ }^{25-28}$, which call for novel synthetic strategies to their authentication. Whereas for intermetallics ${ }^{29}$ numerous quasicrystalline approximants have been isolated, ATs have remained almost elusive in metal-organic materials. Reticular coordination chemistry harbors the synthetic a

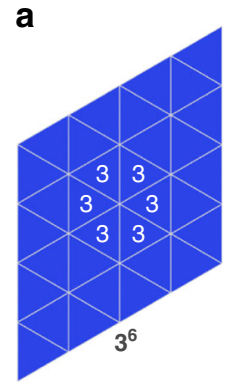

d

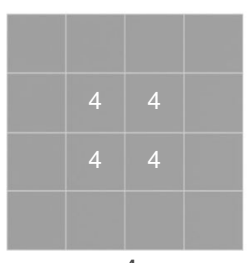

$4^{4}$
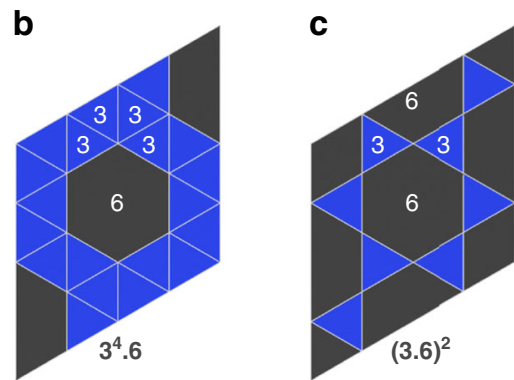

e

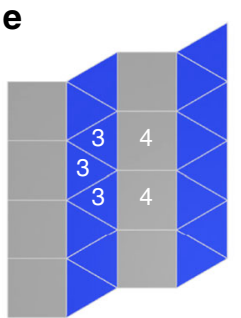

$3^{3} \cdot 4^{2}$ f

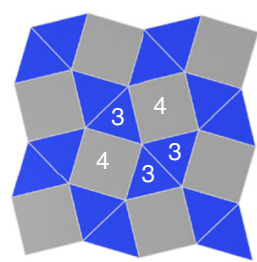

$3^{2} \cdot 4.3 .4$
Fig. 1 Archimedean tessellations. Six of the 11 regular (a, d) and semiregular (b, c, e, f) Archimedean tessellations of the Euclidean plane: a triangular, b snub hexagonal, c trihexagonal (kagomé), d square, e elongated triangular, and $\mathbf{f}$ snub square tiling. handles on controlling dimensionality and topology of metalorganic coordination solids, suitable for targeting complex tessellations. Indeed, applying this strategy resulted in a singular example of a metal-organic quasicrystal consisting of a surface self-assembled array of Eu atom nodes linked by ditopic carbonitrile bridging ligands ${ }^{7}$. This network is reminiscent of a random tiling containing nanoscale motifs of the semi-regular ATs. The flexibility and the large atomic radii of Eu allowed for coordinating up to six ligands in the equatorial plane allowing the formation of local dodecagonal symmetry ${ }^{7}$. Notably, small differences in the preparation conditions led to the formation of prevailing $3^{3} .4^{2}$ or $3^{2} .4 .3 .4$ tilings, which underlines the close relationship of the periodic approximants with the dodecagonal phase. Accordingly, the assembly of atomic $\mathrm{Ce}$ and the same ditopic ligand on a $\operatorname{Ag}(111)$ surface lead to the formation of a $3^{2} .4 .3 .4$ tiling $^{30}$. Only recently, an ionic liquid-based approach led to the isolation of a bulk coordination solid containing layers of $\left[\left(\mathrm{UO}_{2}\right)(1,2,4 \text {-triazolate })_{2.5}\right]^{(1 / 2)-}$, constituting the first example of an $3^{2}$.4.3.4 tiling realized through fivefold coordinated trans$\left\{\mathrm{UO}_{2}\right\}^{2+}$ nodes $^{20}$. Herein, however, the presence of a net charge of the 2D layer could impede the possibilities for further processing of this material for nanostructuring and the nonmagnetic uranyl nodes hinder the exploration of magnetic properties. Similar to uranium, the lanthanide ion series distinguishes itself by a strong propensity to high coordination numbers. In addition, the lanthanide ions exhibit paramount plasticity in their chemical bonding and unique optical and magnetic properties, of immense technological importance, which are not paralleled by any other elements $^{31}$. Motivated by the possibilities to extend these properties to ATs and quasiperiodic materials, we, herein, report a coordination-assembly strategy and the crystallographic characterization of the first example of a lanthanide-based AT in a bulk, molecule-based material.

\section{Results}

Structural characterization and description. Numerous examples of first-row transition element-based 2D coordination solids pillared on $4,4^{\prime}$-bipyridine (bipy) and related ditopic, organic ligands are known ${ }^{32}$. Therein, the limiting, prevailing coordination number of six imposes a square $\left(4^{4}\right.$; cf. Fig. $\left.1 \mathrm{~d}\right)$ tessellation of the $2 \mathrm{D}$ plane calling for employment of significantly larger metal ions for the generation of ATs. The tendency to high coordination numbers in di- and trivalent lanthanide $(\mathrm{Ln}(\mathrm{II} / \mathrm{III}))$ compounds offers the possibility of generating, for instance, $\left.\left[\mathrm{LnI}_{2} \text { (pyridine }\right)_{5}\right]^{+/ 0}\left(\mathrm{Ln}=\mathrm{Lu}^{33}, \mathrm{Sm}^{34}\right)$. Notably, these systems closely resemble a local $D_{5 \mathrm{~h}}$ coordination geometry of the $\mathrm{Ln}$ (II/ III) with the organic ligands placed in the equatorial plane, presumably directed by the space-filling, axial iodide ligands. Translating this structure-directing motif to $2 \mathrm{D}$ coordination solids seems key for tailoring metal-organic ATs and quasicrystals. Indeed, the self-assembly reaction of ytterbium(II) iodide with bipy in acetonitrile at room temperature under strictly anaerobic conditions yielded dark blue, block-shaped crystals of $\mathrm{YbI}_{2}$ (bipy) $)_{2.5} \cdot 1.5 \mathrm{CH}_{3} \mathrm{CN}$ (1) suitable for single-crystal X-ray diffraction analysis. Compound $\mathbf{1}$ crystallizes in the triclinic space group $P \overline{1}$ hosting a single $\mathrm{Yb}$ in the asymmetric unit (Fig. 2 and Supplementary Fig. 1). The $\left\{\mathrm{YbN}_{5}\right\}$ moiety deviates marginally from planarity with a maximal distortion from ideality of $\sim 5 \%$ for $\mathrm{I} 2-\mathrm{Yb}-\mathrm{N} 3$ and approximates closely a $D_{5 \mathrm{~h}}$ coordination environment with $\mathrm{N}-\mathrm{Yb}-\mathrm{N}$ angles in the range from $69^{\circ}$ to $77^{\circ}$, close to the ideal angle of $72^{\circ}$ (Fig. 2b). Within the $2 \mathrm{D}$ plane, the linking of bipy and $\mathrm{Yb}(\mathrm{II})$ forms both square and triangular tiles with the bipy linkers spanning the edges. The squares and triangles closely approximate equilateral geometries with edge lengths of $12.14 \AA$ and $12.25 \AA$ for the squares and $12.32 \AA$, 
a

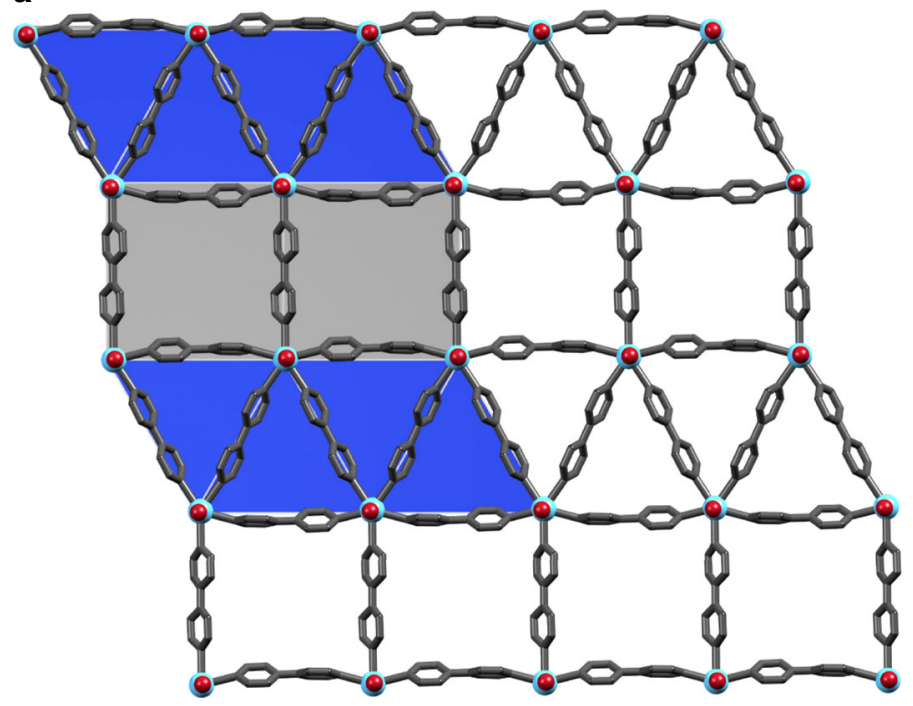

b

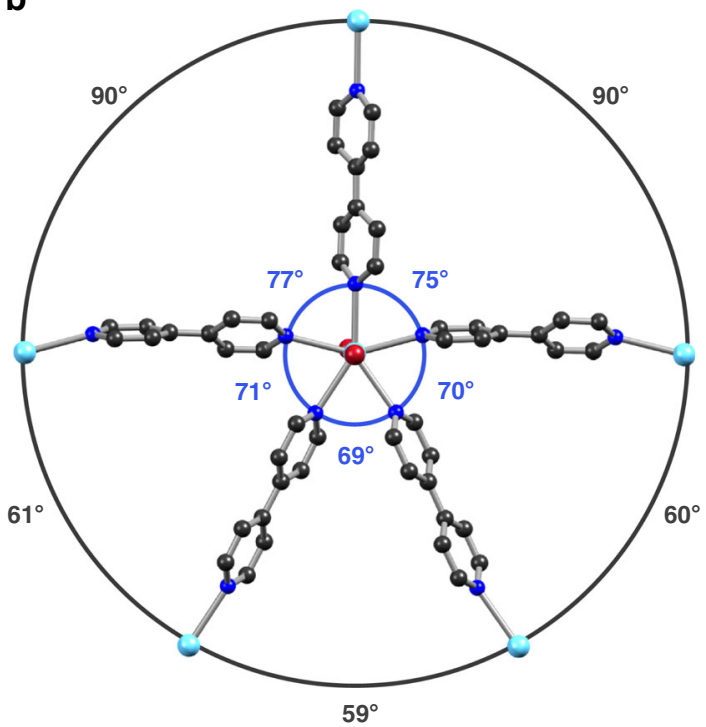

Fig. 2 Structure of 1. Representations of the crystal structure of $\mathbf{1}$ as determined by single-crystal X-ray diffraction at $T=120(1) \mathrm{K}$. a Fragment of the constituent coordination layers with an overlay of the ideal $3^{3} \cdot 4^{2}$ tessellation. $\mathbf{b}$ The five-vertex node of trans- $\left\{\mathrm{Ybl}_{2}\right\}$ and bipy linkers in $\mathbf{1}$. The $\angle \mathrm{N}-\mathrm{Yb}-\mathrm{N}$

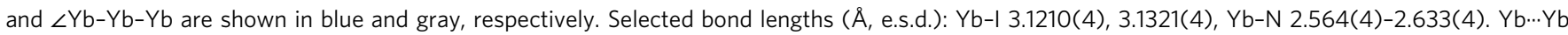
separations $(\AA)$ amount to 12.14 and 12.25 for the squares, and 12.32, 12.14, and 12.34 for the triangles (Yb light blue; I dark red; $\mathrm{N}$ blue; $\mathrm{C}$ gray; hydrogen atoms and co-crystallized $\mathrm{CH}_{3} \mathrm{CN}$ have been omitted for clarity).

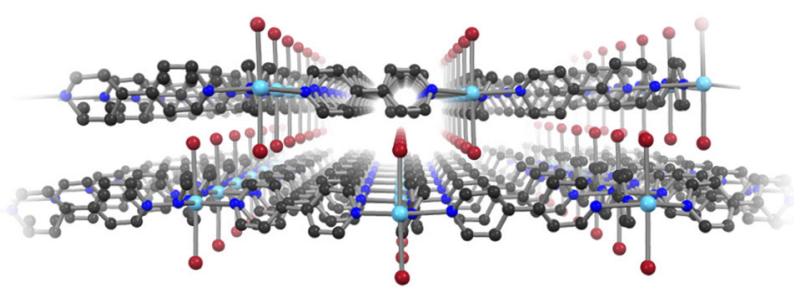

Fig. 3 Perspective view of the 2D nature of 1. Layers of $\mathbf{1}$ in which the iodide ligands point towards the cavities of the adjacent layers. $\angle \mathrm{I}-\mathrm{Yb}-\mathrm{I}$ $175.60(1)^{\circ}$. (Yb, light blue; I, dark red; N, blue; C, gray; hydrogen atoms and co-crystallized $\mathrm{CH}_{3} \mathrm{CN}$ have been omitted for clarity).

$12.14 \AA$, and $12.34 \AA$ for the triangles. Despite the local $D_{5 \mathrm{~h}}$ symmetry, a slight bending of the bipy linkers leads to the formation of an ideal $3^{3} \cdot 4^{2}$ tessellation (Fig. 2a). The $2 \mathrm{D}$ layers are stacked with an inter-plane separation of $\sim 6 \AA$ imposed by the axial iodide ligands (Fig. 3).

The packing motif of the $2 \mathrm{D}$ layers leads to the formation of solvent-accessible pore channels of distorted $3^{3} .4^{2}$ topology along both the crystallographic $a$ and $b$ directions and of distorted $3^{6}$ topology along the $c$ direction (Supplementary Fig. 2). To our knowledge, 1 is the first example of any coordination solid constructed from divalent lanthanide ions. The presence of potentially reducible bipy and strongly reducing $\mathrm{Yb}(\mathrm{II})\left(E^{\ominus} \mathrm{Yb}^{3+} / \mathrm{Yb}^{2+}=-1.1 \mathrm{~V}\right)$ could be expected to lead to the occurrence of redox events, similar to what is found in the reaction between elemental sodium and bipy ${ }^{35}$. However, the single-crystal structure revealed $\mathrm{Yb}-\mathrm{N}$ bond lengths between 2.564(4) $\AA$ and 2.633(4) $\AA$ and Yb-I bond lengths of $3.1210(4) \AA$ and $3.1321(4) \AA$, which are close to those found in the related $\left.\left[\mathrm{Yb}^{\mathrm{II}} \mathrm{I}_{2} \text { (3,5-lutidine) }\right)_{4}\right]$ (Yb-N 2.586(3) $\AA$, Yb-I 3.132 (1) $\AA)^{36}$, and $\left[\mathrm{Yb}^{\mathrm{II}} \mathrm{I}_{2}\left(\mathrm{CH}_{3} \mathrm{CN}\right)_{5}\right](\mathrm{Yb}-\mathrm{N} 2.537(2)-2.566(3) \AA$, $\mathrm{Yb}-\mathrm{I} 3.1124(2), 3.1326(2) \AA)^{37}$, and much further away from $\left.\left[\mathrm{Yb}^{\mathrm{III}} \mathrm{I}_{2} \text { (pyridine) }\right)_{5}\right] \mathrm{I} \cdot 1 / 2$ pyridine $(\mathrm{Yb}-\mathrm{N} 2.454(4)-2.482(3) \AA$, Yb-I 2.9645(3) 2.9729(3) A $)^{38}$.

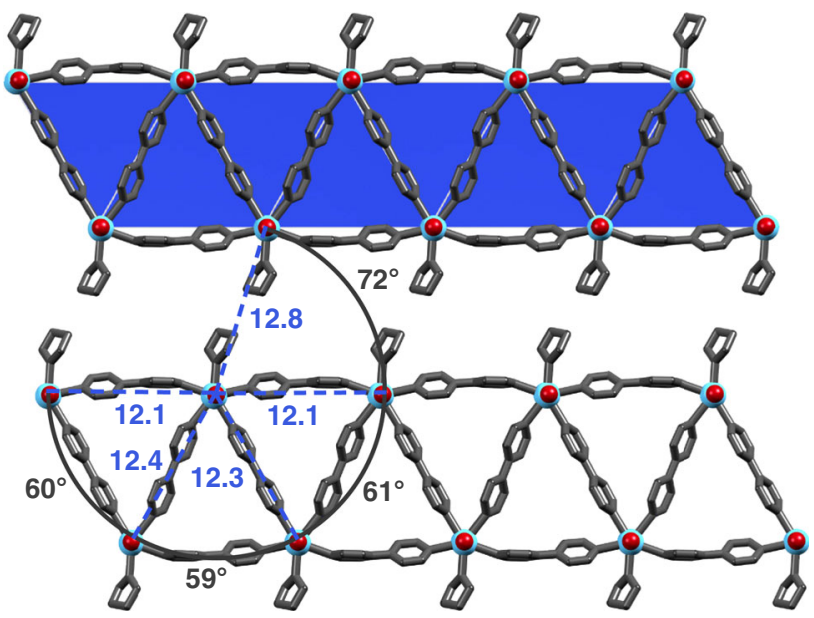

Fig. 4 Structure of 2. Fragment of the structure of $\mathbf{2}$ as determined by single-crystal $\mathrm{X}$-ray diffraction with indicated $\mathrm{Yb} \cdots \mathrm{Yb}$ separation lengths $(\AA)$ and $\angle \mathrm{Yb} \cdots \mathrm{Yb} \cdots \mathrm{Yb}$ angles. Selected additional bond lengths ( $\AA$, e.s.d.) and angles ( ${ }^{\circ}$, e.s.d): $Y b-I$ 3.1311(8), 3.1038(8); Yb-N 2.585(5)-2.666(5); Yb-O 2.454(5); $\angle \mathrm{N}-\mathrm{Yb}-\mathrm{N}$ 69.0(2)-71.9(2); $\angle \mathrm{N}-\mathrm{Yb}-\mathrm{O}$ 73.7(2)-74.1(2). (Yb, light blue; I, dark red).

The structural data thus echo the presence of $\mathrm{Yb}(\mathrm{II})$ and the absence of any $\mathrm{Yb}(\mathrm{III})-$ bipy $^{--}$valence tautomer formation, which has been observed in related, molecular $\mathrm{Yb}(\mathrm{II})$ complexes of $2,2^{\prime}$-bipyridine ${ }^{39,40}$. Changing the solvent of the synthesis to tetrahydrofuran (THF) resulted in a slightly different compound, $\mathrm{YbI}_{2}(\text { bipy })_{2}$ (THF).1.5 THF (2). The dark green 2 is structurally related to 1 but lacks the square tiles due to coordination of a THF solvent molecule and can be considered to be composed of isolated strands of a bisected $3^{6}$ tessellation (Figs. 1a and 4, and Supplementary Fig. 3). Notably, the crystal packing enforces a supramolecular distorted $3^{3} \cdot 4^{2}$ tessellation with interchain $\mathrm{Yb} \cdots \mathrm{Yb} \cdots \mathrm{Yb}$ angles of $72^{\circ}$, and similar inter- and intrachain 
$\mathrm{Yb} \cdots \mathrm{Yb}$ separations (12.8 $\mathrm{\text {vs. }} \sim 12.2 \AA$; Fig. 4). Interestingly, considering only the $\mathrm{Yb}$ sites, the structure can be described as resting at an intermediate position between the $3^{3} \cdot 4^{2}$ (with quadratic tiles: $\left.\angle \mathrm{Yb} \cdots \mathrm{Yb} \cdots \mathrm{Yb}=90^{\circ}\right)$ and the $3^{6}(\angle \mathrm{Yb} \cdots \mathrm{Yb} \cdots \mathrm{Yb}=$ $\left.60^{\circ}\right)$ tessellations. The $\mathrm{Yb}-\mathrm{I}(3.1311(8), 3.1038(8) \AA \AA$ ) and $\mathrm{Yb}-\mathrm{N}$ (2.585(5)-2.666(5) $\AA$ ) bond lengths are all close to those of $\mathbf{1}$, indicating the presence of divalent $\mathrm{Yb}$ in 2 . The oxidation state assignment of $\mathrm{Yb}(\mathrm{II})$ and the absence of any valence-tautomerism in $\mathbf{1}$ and $\mathbf{2}$ were further corroborated by bulk magnetometry. $\mathrm{Yb}$ (II), having a closed-shell [Xe] $4 \mathrm{f}^{14}$ electronic configuration, is expected to be fully diamagnetic. Indeed, the magnetic susceptibility-temperature products, $\chi T$, of both $\mathbf{1}$ and $\mathbf{2}$ at 273 $\mathrm{K}$ were typically vanishing at $\sim 0.06 \mathrm{~cm}^{3} \mathrm{~K} \mathrm{~mol}^{-1}$ (Supplementary Fig. 4). Assuming the identity of a paramagnetic impurity as $\mathrm{Yb}$ (III) $\left(2.57 \mathrm{~cm}^{3} \mathrm{~K} \mathrm{~mol}^{-1}\right.$ calculated for ${ }^{2} \mathrm{~F}_{7 / 2}$ and $\left.g_{J}=8 / 7\right)$, its concentration is estimated to be $<2 \%$.

\section{Discussion}

A quasicrystalline tiling consisting solely of squares and triangles has an optimal square-to-triangle ratio of $\sqrt{ } 3 / 4 \approx 0.43^{8,41}$. Hence, to approach a dodecagonal quasicrystalline tiling with the $3^{3} \cdot 4^{2}$ tessellation structure at hand, triangle-rich motifs need to be introduced into the tiling. The lanthanide ions here stand out as ideal materials modules due to their exceptional structural versatility that is inimitable amongst the inner and outer transition metal elements of the periodic table. This allows their accommodation into the in-plane tetra-, penta-, and hexa-coordinated nodes that coexist in dodecagonal quasicrystals 7,12 . It is here particularly important that the strategy is not reserved for divalent lanthanide ions. Indeed, several molecular complexes of the trivalent lanthanide ions possess an approximate $D_{5 \mathrm{~h}}$ (e.g., $\left[\operatorname{LnI}_{2}(\mathrm{THF})_{5}\right]^{+42-47}$ and $\left[\operatorname{LuI}_{2}(\text { pyridine })_{5}\right]^{+33,38}$ ) coordination environment necessary for their incorporation into such materials. Notably, contrasting the intermetallics, the typical optical transparency of lanthanide-based coordination solids provides for exploitation of optical properties of complex tilings. For instance, ATs offer the potential of photonic band gap opening ${ }^{23,48}$, which in conjunction with the unique luminescence characteristics of the lanthanide ions ${ }^{31}$, lays the foundation for materials with novel optical properties. In addition, the field of molecular magnetism has recently seen a blossoming of approximate $C_{5}$ symmetric single-ion magnets with exceedingly high magnetization reversal barriers ${ }^{49-51}$. Linking of single-ion magnets or lanthanide-based qubits $^{52}$ into ATs and quasicrystals to yield spin-frustrated arrays would be an attractive extension of this work ${ }^{53}$. This approach also comprises controlling metal-ligand redox events with the aim of introducing electronic conductivity and increasing the strength of inter-ion magnetic interactions in ATs and derived network structures as has been developed for regular tessellations with lighter first-row transition element analogs 54 .

In conclusion, we have reported a chemical strategy to tailor AT topologies in coordination solids by employing the propensity for high coordination numbers intrinsic to the lanthanide series ions. The appearance of five-vertex tilings suggests the possibility for the occurrence of metal-organic quasicrystalline phases in a bulk material. Exemplified by the structure of 2, our approach opens up a novel route to design supramolecular metal-organic AT analogs, which is currently pursued for fully organic materials ${ }^{55-57}$. In addition, the $2 \mathrm{D}$ nature hosts potential for nanostructuring and exfoliation of complex tilings, as is now intensively practiced for the $3 \mathrm{~d}$ congeners with regular tessellation structures ${ }^{58}$. Although nanoscale arrays of lanthanide-based ATs have previously been observed on surfaces using scanning tunneling microscopy ${ }^{30}$, the isolation of $\mathbf{1}$ demonstrates the possibilities to design lanthanide-based ATs in bulk, crystalline materials. This result paves the way for next-generation materials with complex and non-periodic tiling structures hosting novel photonic and magnetic phenomena originating from the unique physical properties of the lanthanide ions.

\section{Methods}

Synthesis. All procedures were carried out in an InertLab glovebox with a dry Ar atmosphere. Dry and air-free solvents were obtained from a Puresolv MD 7 solvent purification system. $\mathrm{YbI}_{2}$ and 4,4'-bipyridine (bipy) were supplied by SigmaAldrich and were used as received. Elemental analyses were performed by the Mikroanalytisches Laboratorium Kolbe (Oberhausen, Germany). Synthesis of $\mathrm{YbI}_{2}(\text { bipy })_{2.5} \cdot 1.5 \mathrm{CH}_{3} \mathrm{CN}(\mathbf{1}): \mathrm{YbI}_{2}$ powder $(160 \mathrm{mg}, 375 \mu \mathrm{mol})$ was placed in a standard test tube $(160 \times 16 \mathrm{~mm}$, soda glass). The solid was carefully covered with acetonitrile $(18 \mathrm{~mL})$ with a pipette, as to not swirl up the $\mathrm{YbI}_{2}$. An acetonitrile solution $(3 \mathrm{~mL})$ of bipy $(293 \mathrm{mg}, 1.88 \mathrm{mmol}$ ) was slowly layered on top of that and the reaction mixture was left undisturbed for 7 days. Dark blue crystals formed at the bottom of the reaction tube were isolated using a pipette and carefully washed with cold acetonitrile $\left(-20^{\circ} \mathrm{C} ; 2 \times 5 \mathrm{~mL}\right)$. Yield: $210 \mathrm{mg}(65 \%$ based on $\mathrm{Yb})$. Calculated elemental analysis (found) for $\mathrm{C}_{25} \mathrm{H}_{20} \mathrm{YbI}_{2} \mathrm{~N}_{5}, \mathrm{C}_{3} \mathrm{H}_{4.5} \mathrm{~N}_{1.5}$ : C, $38.26 \%$ (36.67\%); H, 2.81\% (2.63\%); I 28.88\% (28.79\%); N, 10.36\% (9.51\%). Synthesis of $\mathrm{YbI}_{2}(\text { bipy })_{2}$ (THF).1.5 THF (2): $\mathrm{YbI}_{2}(108 \mathrm{mg}, 253 \mu \mathrm{mol})$ and bipy $(216 \mathrm{mg}, 1.38$ mmol) were separately dissolved in THF $(3.7 \mathrm{~mL}$ and $1.3 \mathrm{~mL}$, respectively) and the $\mathrm{YbI}_{2}$ solution was added dropwise to the bipy solution. Afterwards the mixture was kept at $-20^{\circ} \mathrm{C}$ for 2 days. The dark green crystals formed at the bottom of the vial were filtered and washed with toluene $(10 \mathrm{~mL})$ and dried under vacuum. Yield: $177 \mathrm{mg}$ (70\% based on $\mathrm{Yb}$ ). Calculated elemental analysis (found) for $\mathrm{YbI}_{2} \mathrm{C}_{24} \mathrm{H}_{24} \mathrm{~N}_{4} \mathrm{O}_{1}$, $\mathrm{C}_{6} \mathrm{H}_{12} \mathrm{O}_{1.5}: \mathrm{C}, 39.19 \%$ (37.95\%); H, 3.95\% (3.66\%); I 27.60\% (28.59\%); N, 6.09\% $(6.35 \%)$.

Crystallography. Single crystals of $\mathbf{1}$ and $\mathbf{2}$ were covered with polybutene oil (Aldrich, $>90 \%$ ) and mounted onto a nylon loop, which was attached to a SuperNova Dual Source CCD diffractometer. Data were collected using Mo Ka radiation at $T=120(1) \mathrm{K}$. Using Olex $2^{59}$, the structure was solved with the ShelXT ${ }^{60}$ structure solution program and refined with the SHELXS ${ }^{61}$ refinement package using least squares minimization. All non-hydrogen atoms were refined anisotropically (cf. Supplementary Table 1). The crystal structure of $\mathbf{1}$ consists of one additional, highly disordered solvent molecule in the unit cell, i.e., half a solvent molecule per formula unit, which could not be modeled accurately and was treated by solvent mask method implemented in Olex ${ }^{62}$. Twenty-three electrons were found in a volume of $282 \AA^{3}$ in one void per unit cell. This indicates the presence of one acetonitrile molecule per unit cell, which accounts for 22 electrons. Similarly, a solvent mask was calculated for $\mathbf{2}$ and 38 electrons were found in a volume of $177 \AA^{3}$ in one void per unit cell. This is consistent with the presence of one molecule of THF per unit cell, which accounts for 40 electrons. Powder X-ray diffraction patterns (Supplementary Fig. 5) were measured at room temperature in transmission mode with a Huber $\mathrm{G} 670$ powder diffractometer using $\mathrm{Cu} \mathrm{Ka} \alpha_{1}(\lambda=$ $1.5406 \AA$, quartz monochromator) radiation. The powder samples were measured in sealed bags and the powder of $\mathbf{1}$ was additionally immersed in polybutene oil (Aldrich, $>90 \%$ ) to suppress loss of co-crystallized $\mathrm{CH}_{3} \mathrm{CN}$ molecules. The resulting powder patterns were background corrected.

Magnetization measurements. The isofield dc magnetization measurements were performed using the VSM option on a QuantumDesign Dynacool Physical Property Measurement System equipped with a 9T dc magnet in the temperature range from $1.7 \mathrm{~K}$ to $273 \mathrm{~K}$ in a magnetic field of $0.1 \mathrm{~T}$. The polycrystalline samples were loaded into standard QuantumDesign powder capsules inside an Ar-filled glovebox. The experimental data were corrected for diamagnetic contributions from the sample holder and the intrinsic sample diamagnetism.

\section{Data availability}

The X-ray crystallographic coordinates for structures reported in this Article have been deposited at the Cambridge Crystallographic Data Centre (CCDC), under the deposition numbers CCDC 1988173-1988174. These data can be obtained free of charge from The Cambridge Crystallographic Data Centre via www.ccdc.cam.ac.uk/data_request/cif. All other relevant data are available from the authors on request.

Received: 11 March 2020; Accepted: 17 August 2020; Published online: 17 September 2020

\section{References}

1. Steurer, W. Quasicrystals: what do we know? What do we want to know? What can we know? Acta Crystallogr. A Found Adv. A74, 1-11 (2018).

2. Dubois, J. -M. Properties- and applications of quasicrystals and complex metallic alloys. Chem. Soc. Rev. 41, 6760-6777 (2012). 
3. Zoorob, M. E., Charlton, M. D. B., Parker, G. J., Baumberg, J. J. \& Netti, M. C. Complete photonic bandgaps in 12-fold symmetric quasicrystals. Nature $\mathbf{4 0 4}$ 740-743 (2000).

4. Deguchi, K. et al. Quantum critical state in a magnetic quasicrystal. Nat. Mater. 11, 1013-1016 (2012)

5. Vardeny, Z. V., Nahata, A. \& Agrawal, A. Optics of photonic quasicrystals. Nat. Photonics 7, 177-187 (2013).

6. Shechtman, D., Blech, I., Gratias, D. \& Cahn, J. W. Metallic phase with longrange orientational order and no translational symmetry. Phys. Rev. Lett. 53, 1951-1953 (1984).

7. Urgel, J. I. et al. Quasicrystallinity expressed in two-dimensional coordination networks. Nat. Chem. 8, 657-662 (2016).

8. Leung, P. W., Henley, C. L. \& Chester, G. V. Dodecagonal order in a twodimensional Lennard-Jones system. Phys. Rev. B 39, 446-458 (1989).

9. Oxborrow, M. \& Henley, C. L. Random square-triangle tilings: a model for twelvefold-symmetric quasicrystals. Phys. Rev. B 48, 6966-6998 (1993).

10. Ishimasa, T., Nissen, H.-U. \& Fukano, Y. New ordered state between crystalline and amorphous in Ni-Cr particles. Phys. Rev. Lett. 55, 511-513 (1985).

11. Chen, H., Li, D. X. \& Kuo, K. H. New type of two-dimensional quasicrystal with twelvefold rotational symmetry. Phys. Rev. Lett. 60, 1645-1648 (1988).

12. Xiao, C., Fujita, N., Miyasaka, K., Sakamoto, Y. \& Terasaki, O. Dodecagonal tiling in mesoporous silica. Nature 487, 349-353 (2012).

13. Talapin, D. V. et al. Quasicrystalline order in self-assembled binary nanoparticle superlattices. Nature 461, 964-967 (2009).

14. Hayashida, K., Dotera, T., Takano, A. \& Matsushita, Y. Polymeric quasicrystal: Mesoscopic quasicrystalline tiling in $A B C$ star polymers. Phys. Rev. Lett. 98, 195502 (2007)

15. Frank, F. C. \& Kasper, J. S. Complex alloy structures regarded as sphere packings. II. Analysis and classification of representative structures. Acta Cryst. 12, 483-499 (1959).

16. Grünbaum, B. \& Shephard, G. C. Tilings by regular polygons. Math. Mag. 50, 227-247 (1977).

17. Pearce, P. Structure in Nature is a Strategy for Design (MIT Press, 1978).

18. Yamamoto, A. Crystallography of quasiperiodic crystals. Acta Cryst. A52, 509-560 (1996).

19. Steurer, W. Twenty years of structure research on quasicrystals. Part I. Pentagonal, octagonal, decagonal and dodecagonal quasicrystals. $Z$. Kristallogr. Cryst. Mater. 219, 391-446 (2004).

20. Smetana, V., Kelley, S. P., Mudring, A. -V. \& Rogers, R. D. A fivefold $\mathrm{UO}_{2}{ }^{2+}$ node is a path to dodecagonal quasicrystal approximants in coordination polymers. Sci. Adv. 6, eaay7685 (2020).

21. Poeppelmeier, K. R. \& Azuma, M. A new spin on frustration. Nat. Chem. 3, 758-759 (2011).

22. David, S., Chelnokov, A. \& Lourtioz, J.-M. Isotropic photonic structures: Archimedean-like tilings and quasi-crystals. IEEE J. Quantum Electron. 37, 1427-1434 (2001).

23. Ueda, K., Dotera, T. \& Gemma, T. Photonic band structure calculations of two-dimensional Archimedean tiling patterns. Phys. Rev. B 75, 195122 (2007).

24. Stelson, A. C., Britton, W. A. \& Liddell Watson, C. M. Photonic crystal properties of self-assembled Archimedean tilings. J. Appl. Phys. 121, 023101 (2017).

25. Harrison, A. First catch your hare: the design and synthesis of frustrated magnets. J. Phys. Condens. Matter 16, S553-S572 (2004).

26. Canals, B. et al. Fragmentation of magnetism in artificial kagome dipolar spin ice. Nat. Commun. 7, 1-6 (2016)

27. Yazyev, O. V. An upside-down magnet. Nat. Phys. 15, 424-425 (2019).

28. Yu, U. Ising antiferromagnet on the Archimedean lattices. Phys. Rev. E 91, 062121 (2015)

29. O’Keefe, M. \& Hyde, B. G. Plane nets in crystal chemistry. Philos. Trans. $R$ Soc. A 295, 553-618 (1980).

30. Écija, D. et al. Five-vertex Archimedean surface tessellation by lanthanidedirected molecular self-assembly. Proc. Natl Acad. Sci. USA 110, 6678-6681 (2013).

31. Bünzli, J.-C. G. Lanthanide photonics: shaping the nanoworld. Trends Chem. 1, 751-762 (2019)

32. Noro, $\mathrm{S}$. et al. Framework engineering by anions and porous functionalities of $\mathrm{Cu}$ (II)/4,4'-bpy coordination polymers. J. Am. Chem. Soc. 124, 2568-2583 (2002).

33. Giesbrecht, G. R., Gordon, J. C., Clark, D. L. \& Scott, B. L. Auto-ionization in lutetium iodide complexes: Effect of the ionic radius on lanthanide-iodide binding. Inorg. Chem. 43, 1065-1070 (2004).

34. Berthet, J. C., Thuery, P. \& Ephritikhine, M. CCDC 959301: experimental crystal structure determination, https://doi.org/10.5517/CC11676P (2013).

35. Denning, M. S., Irwin, M. \& Goicoechea, J. M. Synthesis and characterization of the $4,4^{\prime}$-bipyridyl dianion and radical monoanion. A structural study. Inorg. Chem. 47, 6118-6120 (2008).

36. Maunder, G. H. \& Sella, A. Simple adducts of samarium and ytterbium diiodide: synthesis and molecular structures of $\mathrm{LnI}_{2}(3,5 \text {-lutidine })_{2}(\mathrm{Ln}=\mathrm{Sm}$, $\mathrm{Yb}$ ) and $\mathrm{YbI}_{2}$ (4-t-butylpyridine) $)_{2}$. Polyhedron 17, 63-68 (1998).
37. Kubus, M., Voigt, L. \& Pedersen, K. S. Pentagonal-bipyramidal acetonitrile complexes of the lanthanide(II) iodides. Inorg. Chem. Commun. 114, 107819 (2020).

38. Huebner, L., Kornienko, A., Emge, T. J. \& Brennan, J. G. Heterometallic lanthanide group 12 metal iodides. Inorg. Chem. 43, 5659-5664 (2004).

39. Schultz, M., Boncella, J. M., Berg, D. J., Tilley, T. D. \& Andersen, R. A. Coordination of 2,2'-bipyridyl and 1,10-phenanthroline to substituted ytterbocenes: an experimental investigation of spin coupling in lanthanide complexes. Organometallics 21, 460-472 (2002).

40. Goudy, V., Jaoul, A., Cordier, M., Clavaguéra, C. \& Nocton, G. Tuning the stability of $\mathrm{Pd}(\mathrm{IV})$ intermediates using a redox non-innocent ligand combined with an organolanthanide fragment. J. Am. Chem. Soc. 139, 10633-10636 (2017).

41. Widom, M. Bethe ansatz solution of the square-triangle random tiling model Phys. Rev. Lett. 70, 2094-2097 (1993).

42. Anfang, S. et al. Synthese und Kristallstrukturen der Seltenerd-Komplexe $\left[\mathrm{LaI}_{2}(\mathrm{THF})_{5}\right]^{+} \mathrm{I}_{3}^{-},\left[\mathrm{SmCl}_{3}(\mathrm{THF})_{4}\right],\left[\mathrm{ErCl}_{2}(\mathrm{THF})_{5}\right]^{+}\left[\mathrm{ErCl}_{4}(\mathrm{THF})_{2}\right]^{-}$, $\left[\mathrm{ErCl}_{3}(\mathrm{DME})_{2}\right]$ und $\left[\mathrm{Na}(18-\mathrm{Krone}-6)(\mathrm{THF})_{2}\right]^{+}\left[\mathrm{YbBr}_{4}(\mathrm{THF})_{2}\right]^{-}$. Z. Anorg Allg. Chem. 623, 1425-1432 (1997).

43. Izod, K., Liddle, S. T. \& Clegg, W. A convenient route to lanthanide triiodide THF solvates. Crystal structures of $\mathrm{LnI}_{3}(\mathrm{THF})_{4}[\mathrm{Ln}=\mathrm{Pr}]$ and $\mathrm{LnI}_{3}(\mathrm{THF})_{3.5}$ $[\mathrm{Ln}=\mathrm{Nd}, \mathrm{Gd}, \mathrm{Y}]$. Inorg. Chem. 43, 214-218 (2004).

44. Evans, W. J. et al. Synthesis and characterization of the samarium-cobalt complexes $\left(\mathrm{C}_{5} \mathrm{Me}_{5}\right)_{2}(\mathrm{THF}) \mathrm{SmCo}(\mathrm{CO})_{4}$ and $\left[\mathrm{SmI}_{2}(\mathrm{THF})_{5}\right]\left[\mathrm{Co}(\mathrm{CO})_{4}\right]$ : $\mathrm{x}$-ray crystal structure of a seven-coordinate samarium(III) cation complex. Inorg. Chem. 24, 4620-4623 (1985).

45. Gompa, T. P. et al. Diethyl ether adducts of trivalent lanthanide iodides. Dalton Trans. 48, 8030-8033 (2019)

46. Fagin, A. A. et al. Iodide-sulfides of dysprosium: elucidation of the pathway to lanthanide iodide-sulfide-nitride clusters. Inorg. Chim. Acta 469, 227-230 (2018)

47. Niemeyer, M. trans-Diiodopentakis(tetrahydrofuran)ytterbium(III) tetraiodotrans-bis(tetrahydrofuran)ytterbium(III). Acta Cryst. E57, m363-m364 (2001).

48. Dotera, T. \& Gemma, T. Dodecagonal quasicrystal in a polymeric alloy. Philos Mag. 86, 1085-1091 (2006).

49. Ding, Y.-S., Chilton, N. F., Winpenny, R. E. P. \& Zheng, Y.-Z. On approaching the limit of molecular magnetic anisotropy: a near-perfect pentagonal bipyramidal dysprosium(III) single-molecule magnet. Angew. Chem. Int. Ed. 55, 16071-16074 (2016).

50. Ding, Y.-S. et al. Field- and temperature-dependent quantum tunnelling of the magnetisation in a large barrier single-molecule magnet. Nat. Commun. 9, 3134 (2018).

51. Yu, K.-X. et al. Enhancing magnetic hysteresis in single-molecule magnets by ligand functionalization. Chem 6, 1777-1793. https://doi.org/10.1016/j. chempr.2020.04.024 (2020).

52. Hussain, R. et al. Coherent manipulation of a molecular Ln-based nuclear qudit coupled to an electron qubit. J. Am. Chem. Soc. 140, 9814-9818 (2018)

53. Graham, M. J., Zadrozny, J. M., Fataftah, M. S. \& Freedman, D. E. Forging solid-state qubit design principles in a molecular furnace. Chem. Mater. 29, 1885-1897 (2017)

54. Pedersen, K. S. et al. Formation of the layered conductive magnet $\mathrm{CrCl}_{2}$ (pyrazine) $)_{2}$ through redox-active coordination chemistry. Nat. Chem. 10, 1056-1061 (2018).

55. Zhang, Y.-Q. et al. Complex supramolecular interfacial tessellation through convergent multi-step reaction of a dissymmetric simple organic precursor. Nat. Chem. 10, 296-304 (2018).

56. Cheng, F. et al. Two-dimensional tessellation by molecular tiles constructed from halogen-halogen and halogen-metal networks. Nat. Commun. 9, 4871 (2018).

57. Beldjoudi, Y. et al. Supramolecular tessellations by a rigid naphthalene diimide triangle. J. Am. Chem. Soc. 141, 17783-17795 (2019).

58. López-Cabrelles, J. et al. Isoreticular two-dimensional magnetic coordination polymers prepared through pre-synthetic ligand functionalization. Nat. Chem. 10, 1001-1007 (2018).

59. Dolomanov, O. V., Bourhis, L. J., Gildea, R. J., Howard, J. A. K. \& Puschmann, H. OLEX2: a complete structure solution, refinement and analysis program. J. Appl. Cryst. 42, 339-341 (2009).

60. Sheldrick, G. M. SHELXT-Integrated space-group and crystal-structure determination. Acta Cryst. A71, 3-8 (2015).

61. Sheldrick, G. M. Crystal structure refinement with SHELXL. Acta Cryst. C71, 3-8 (2015).

62. van der Sluis, P. \& Spek, A. L. BYPASS: an effective method for the refinement of crystal structures containing disordered solvent regions. Acta Cryst. A46, 194-201 (1990).

\section{Acknowledgements}

K.S.P. thanks the VILLUM Foundation for a VILLUM Young Investigator grant (15374) and the Carlsberg Foundation (CF-17-0637), the Brdr. Hartmanns foundation, and the Torkil Holm foundation for research infrastructure grants. Drs. R. Clérac and P. Perlepe are thanked for preliminary magnetic measurements. 


\section{Author contributions}

L.V. and K.S.P. formulated the project. L.V. synthesized and characterized the compounds. L.V. and M.K. collected and analyzed the X-ray diffraction data. All authors contributed to writing the manuscript.

\section{Competing interests}

The authors declare no competing interests.

\section{Additional information}

Supplementary information is available for this paper at https://doi.org/10.1038/s41467020-18328-5.

Correspondence and requests for materials should be addressed to K.S.P.

Peer review information Nature Communications thanks Steven Kelley and other, anonymous, reviewers for their contributions to the peer review of this work.

Reprints and permission information is available at http://www.nature.com/reprints
Publisher's note Springer Nature remains neutral with regard to jurisdictional claims in published maps and institutional affiliations.

\section{(c) (1)}

Open Access This article is licensed under a Creative Commons Attribution 4.0 International License, which permits use, sharing, adaptation, distribution and reproduction in any medium or format, as long as you give appropriate credit to the original author(s) and the source, provide a link to the Creative Commons license, and indicate if changes were made. The images or other third party material in this article are included in the article's Creative Commons license, unless indicated otherwise in a credit line to the material. If material is not included in the article's Creative Commons license and your intended use is not permitted by statutory regulation or exceeds the permitted use, you will need to obtain permission directly from the copyright holder. To view a copy of this license, visit http://creativecommons.org/ licenses/by/4.0/.

(c) The Author(s) 2020 